\title{
Predicting the Visual Focus of Attention in Multi-Person Discussion Videos
}

\author{
Chongyang Bai $^{1 *}$, Srijan Kumar ${ }^{2,3}$, Jure Leskovec ${ }^{2}$, Miriam Metzger ${ }^{4}$, \\ Jay F. Nunamaker ${ }^{5}$ and V.S. Subrahmanian ${ }^{1}$ \\ ${ }^{1}$ Dartmouth College \\ ${ }^{2}$ Stanford University \\ ${ }^{3}$ Georgia Institute of Technology \\ ${ }^{4}$ University of California Santa Barbara \\ ${ }^{5}$ University of Arizona \\ cy@cs.dartmouth.edu, \{srijan,jure\}@cs.stanford.edu, \\ metzger@ucsb.edu,jnunamaker@cmi.arizona.edu,vs@dartmouth.edu
}

\begin{abstract}
Visual focus of attention in multi-person discussions is a crucial nonverbal indicator in tasks such as inter-personal relation inference, speech transcription, and deception detection. However, predicting the focus of attention remains a challenge because the focus changes rapidly, the discussions are highly dynamic, and the people's behaviors are inter-dependent. Here we propose ICAF (Iterative Collective Attention Focus), a collective classification model to jointly learn the visual focus of attention of all people. Every person is modeled using a separate classifier. ICAF models the people collectively - the predictions of all other people's classifiers are used as inputs to each person's classifier. This explicitly incorporates interdependencies between all people's behaviors. We evaluate $I C A F$ on a novel dataset of 5 videos (35 people, 109 minutes, 7604 labels in all) of the popular Resistance game and a widely-studied meeting dataset with supervised prediction. ICAF outperforms the strongest baseline by $1 \%-5 \%$ accuracy in predicting the people's visual focus of attention. Further, we propose a lightly supervised technique to train models in the absence of training labels. We show that light-supervised $I C A F$ performs similar to the supervised $I C A F$, thus showing its effectiveness and generality to previously unseen videos.
\end{abstract}

\section{Introduction}

Given a group $G$ of people, a person $P \in G$, and a short video clip $v(1 / 3 \mathrm{rd} \mathrm{sec})$, the Visual Focus of Attention (VFOA) problem is to automatically predict who person $P$ is looking at among all people in $G$ in the video clip $v$. Solving the VFOA problem can provide profound insights into a number of factors, e.g., who is the dominant person in the group [Hall et al., 2005], who supports/opposes who in the group, who trusts/distrusts who in the group [Knapp et al., 2013].

\footnotetext{
${ }^{*}$ Contact Author
}

Figure 1(a) illustrates some of the challenges involved. First, even within a very short 1 second clip, a person may look at many people. The four frames shown in Figure 1(a) show the pictured subject looking at three people. Second, multi-person discussions are highly dynamic because many people may speak at the same time and the speakers change rapidly (Figure 1) — and as people often look at a speaker, solving VFOA requires the ability to rapidly estimate the VFOA. Third, non-verbal behaviors (e.g. eye rolling, head shaking) of people may influence another person's VFOA. Returning to Figure 1(a), one would expect people to look at the lady shown when she is speaking - however, their gaze may turn elsewhere if some unseen person makes a gesture. Alternatively, predicting the VFOA of person $\mathrm{P}$ might depend on predicting the VFOA of person $\mathrm{P} 1$ as both of them might be looking at the same person P2 who is speaking or gesturing. In short, solving VFOA requires reasoning at the subsecond level and making rapid changes that take into account not only video of the person $P$ whose gaze we are trying to predict, but also that of others.

We address these challenges via a novel algorithm called ICAF (stands for Iterative Collective Attention Focus) which: (i) reasons at the $1 / 3$ second level that prior research has established as the normal duration humans need to visually focus their attention [Rayner, 2009], (ii) incorporates collective classification [Sen et al., 2008; Kong et al., 2012] intuitions to capture the fact that where person $P$ is looking might depend on where others are looking, and simultaneously assign VFOAs to all people rather than doing so independently, and (iii) ICAF iteratively builds a multi-layer network that captures the evolution of the collective classification. This captures the idea that predictions of who $P$ is looking at depends on predictions of who others in the group are looking at. (iv) ICAF specifically captures the temporal dependency of VFOA, e.g. the conditional probability that $\mathrm{P}$ is looking at $\mathrm{Q}$, given that she was looking at $\mathrm{Q}$ in the previous $1 / 3 \mathrm{sec}$. To the best of our knowledge, no prior work on gaze estimation has considered using where others are currently looking and using this to arrive at a joint prediction as we do.

We introduce a novel dataset (109 mins of video from 5 episodes of the Resistance game in 3 different countries with 


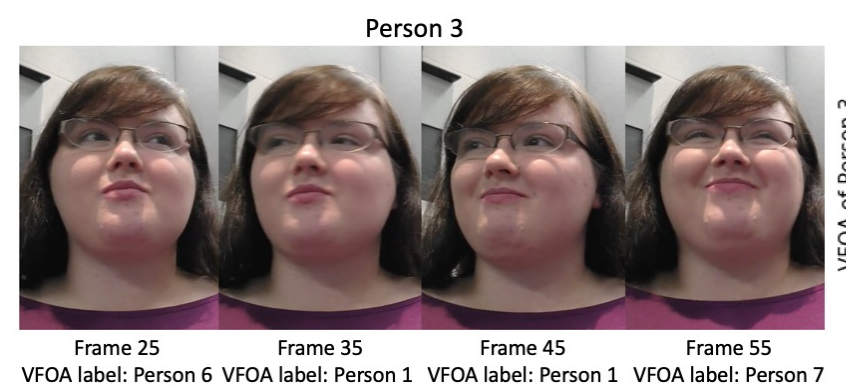

(a)

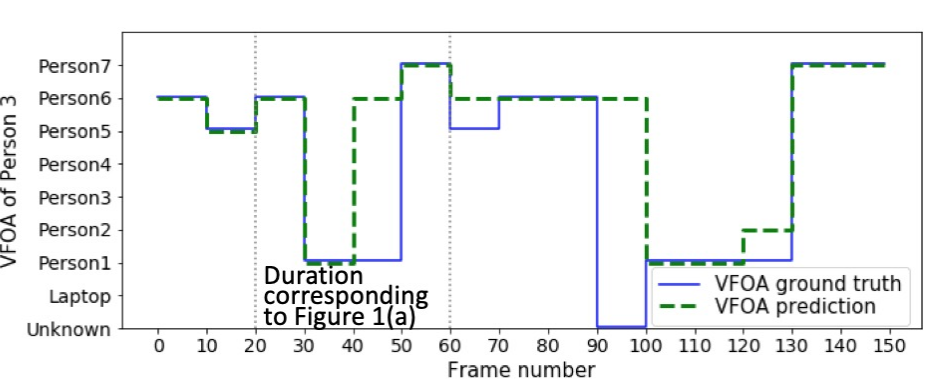

(b)

Figure 1: (a) An example of a person's (Person 3) Visual Focus of Attention (VFOA) in 4 frames out of a contiguous 4/3 second (40 frames) during a discussion. person 3's VFOA changes rapidly within this short time period, from looking at persons 6, 1, 1, 7, in frames 25, 35, 45, and 55, respectively. Note that even though the head pose in frames 25 and 55 are similar, the VFOA is different (6 vs 7) (b) Person 3's ground truth VFOA and predicted VFOA made by the proposed method, ICAF, of a 5-second discussion clip in which frames 20-60 correspond to Figure 1 (a). We observe that $I C A F$ is able to efficiently predict the rapid change in VFOA.

35 people). The data was annotated with ground truth VFOA at the $1 / 3$ second level (a huge task by itself leading to over 19,000 annotated $1 / 3$ second clips). Resistance is an immensely popular, dynamic, animated (and sometimes very noisy) party game involving 5-8 people per game.

We experimentally show that ICAF outperforms several strong baselines in predicting people's next VFOA by over $1.3 \%$, i.e. given a training video up to second $t$, we predict where each person looks at second $t+1 / 3$. Moreover, ICAF outperforms the best baseline between $1 \%-5 \%$ when predicting next $k$ VFOAs. For example Figure 1(b) shows that even though Person 3 rapidly changes her VFOA during a 5 second multi-person discussion, ICAF predicts her VFOA correctly in 11 out of 14 points (78.6\% accuracy). Finally, we experimentally show that both temporal dependency and collective classification boost ICAF's performance.

Since getting ground truth labels is a tedious task, we create a lightly supervised version of ICAF that uses the speaker label to make predictions. We experimentally show that lightly supervised $I C A F$ has similar performance to $I C A F$, showing the potential of using $I C A F$ for previously unseen videos.

The demo, code, and predicted VFOA networks are available at: https://cs.dartmouth.edu/dsail/demos/icaf.

\section{Related Work}

As tracking eye gaze in video is difficult (video resolution, eye visibility, etc.), many estimate head pose as VFOA [Stiefelhagen et al., 1999; Voit and Stiefelhagen, 2008; Zhang et al., 2008; Stiefelhagen and Zhu, 2002]. In real cases, head pose and VFOA may differ. Figure 1(a) shows an example in our dataset-while the person's head pose is similar in frames 25 and 55, her VFOA is different. [Asteriadis et al., 2014] fused head pose and eye gaze to reduce prediction error. Our $I C A F$ additionally adds speaking probabilities as features.

[Ba and Odobez, 2009] used head pose to model VFOA by GMM and HMM with person-based Maximum A Posterior parameters. [Sheikhi and Odobez, 2012] added temporal gaze change in HMM. Their methods predict VFOA individually. Instead, our collective classification model enables joint predictions of all people based on head pose and eye gaze.
In group settings, people's VFOA are influenced by each other. [Stiefelhagen et al., 2002] introduced speaking priors to capture VFOA. [Ba and Odobez, 2008] further used meeting context (e.g slides updating) prior. [Ba and Odobez, 2011] additionally created a Dynamic Bayesian Network capturing the shared VFOA, but the sharing prior is constant and same for all people. In contrast, our ICAF adds inter-person dependency, enabling the classifiers to learn the weights for other inputs, allowing changes over time as behaviors shift during a video. [Massé et al., 2017] proposed a temporal graphical model to jointly track people's gaze and VFOA. Unlike us, they assumed conditional independence of people's VFOAs given their observed head poses. [Duffner and Garcia, 2013; Duffner and Garcia, 2016] clustered VFOA via Histogram of Gradient features. Unlike them, we use a speak prior for light supervision and show its efficacy by comparing with fully supervised results.

Collective classification. Collective classification methods are widely used in graph mining tasks such as node labeling [Sen et al., 2008; Kong et al., 2012], link prediction [Taskar et al., 2004] and a combination of both [Bilgic et al., 2007]. These methods are able to correlate node/edge attributes to train a mutually dependent classifier ensemble. However, none of these models directly predicting VFOA from videos. To the best of our knowledge, ICAF is the first method to use collective classification to predict the VFOA of all people simultaneously in a multi-person video.

\section{Dataset and Problem Setup}

We collected a dataset involving the Resistance game ${ }^{1}$ containing five games from five different locations - three from U.S.A., one from Israel, and one from Singapore. In each game, up to eight people are seated in an octagon layout (Figure 2). It has a total of 35 people whose goal is to identify deceptive people for additional financial reward. Each person has a tablet in front of them which records their activity. At the start of every game, all people introduce themselves, followed by several rounds of discussion

\footnotetext{
${ }^{1}$ https://en.wikipedia.org/wiki/The_Resistance_(game)
} 
Figure 2: Data collection setup

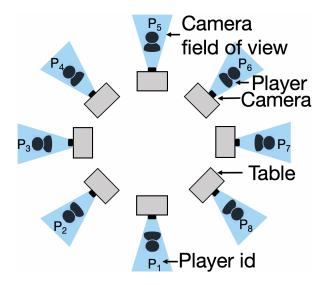

Table 1: Resistance dataset

\begin{tabular}{c|c|c|c}
\hline $\begin{array}{c}\text { Video } \\
\text { id }\end{array}$ & $\begin{array}{c}\text { Number of } \\
\text { seconds }\end{array}$ & $\begin{array}{c}10 \text {-frame } \\
\text { segments }\end{array}$ & $\begin{array}{c}\text { Number of } \\
\text { labels }\end{array}$ \\
\hline 1 & 1062 & 3186 & 1086 \\
2 & 896 & 2688 & 1541 \\
3 & 1435 & 4305 & 1516 \\
4 & 1984 & 5952 & 2060 \\
5 & 1134 & 3402 & 1401 \\
\hline Total & 6511 & 19533 & 7604 \\
\hline
\end{tabular}

where 2-3 people are deceptive and do not want to be identified by the other people whose goal is to unmask them. The people may not leave their seats. The discussions are emergent as there is no pre-determined presenter or leader.

We generated ground-truth labels for people's VFOA for every 10 frames (1/3 seconds in 30 frames per second videos), the time taken to register one's attention [Rayner, 2009]. Figure1(a) is an example. An expert manually assigned one label for every 10 frame segment of each person. For each person, there are eight possible points of focus-one of the other 7 people and the tablet. A label is assigned if the person looks at the object (person or tablet) for the majority of the 10 frames, otherwise, an 'unknown' label is assigned. This results in a total of 7604 valid labeled segments. The 'unknown'-labeled segments are not used for training or testing.

We extract 3 clips from each game-the entire introduction round (where at most one person is speaking at a time), and two 5-second discussions (where multiple people are simultaneously speaking). This gives 6511 seconds of data in total for the 5 games. Table 1 shows the data distribution by game.

AMI corpus. We also used the widely-studied AMI meeting corpus [McCowan et al., 2005], which is highly structured. In this dataset, we used closeup videos of 12 meetings with available VFOA annotation. Each meeting has 4 people and lasts 25 minutes on average. The VOFA targets are 4 people, table, whiteboard and slide screen.

\subsection{Feature Extraction}

We extract two sets of features from the clips: face-based features and speaking probability features. As with face-based features, we extract the person's head pose angles and eye gaze vectors using OpenFace [Baltrusaitis et al., 2018] since the tablet cameras can capture close-up video of each person.

Speaking prediction. We use visual information to predict if a person is speaking at an instance. First, we get 2-dimensional lip contour points $X^{(t)}=\left\{\left(x_{i}^{(t)}, y_{i}^{(t)}\right), i=\right.$ $1, \ldots, n\}$ at frame $t$ from OpenFace and normalize $X^{(t)}$ by its bounding box to avoid the influence of head movement. Second, we compute the gradient of point positions over time to capture mouth movement, which is $\vec{g}_{i}^{(t)}=\left(x_{i}^{(t)}-\right.$ $\left.x_{i}^{(t-1)}, y_{i}^{(t)}-y_{i}^{(t-1)}\right), i=1, \ldots, n$, and aggregate them as a frame feature vector $\vec{g}^{(t)}$. Third, we get feature $G^{(t)}$ by concatenating $\left(\vec{g}^{(t-s+1)}, \vec{g}^{(t-s+2)}, \ldots, \vec{g}^{(t)}, \ldots, \vec{g}^{(t+s)}\right)$ around time $t$, in a window of size $2 s$. This forms a sliding window over time. We use $G^{(t)}$ as a feature, and the introduction part of a game from this dataset to train a general speaking detection model SP. Finally, the speaking probability of a

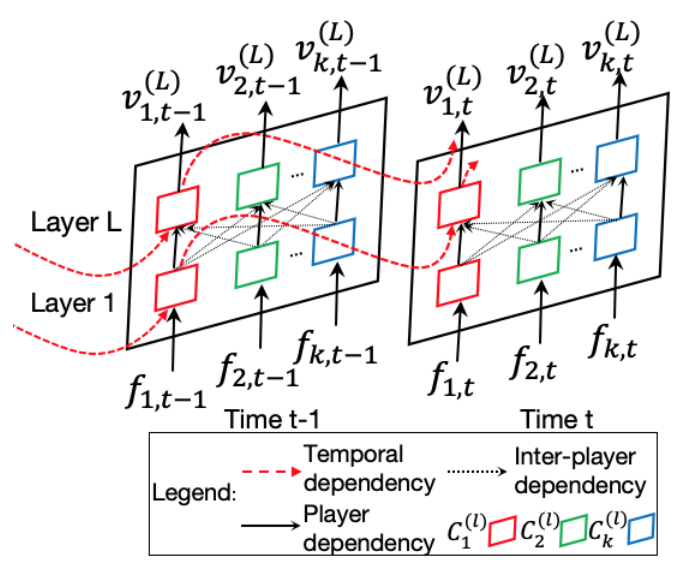

Figure 3: Architecture of the iterative collective classification model, ICAF. Each classifier $C_{i}$ takes three inputs: output of its previous layer (person dependency), previous time (temporal dependency), and other people's output (inter-person dependency). Figure is best viewed in color.

person at time $\mathrm{t}$ is given by $s=\mathbf{S P}\left(G^{(t)}\right)$.

We do not create a new model for head pose angles or eye gaze vector extraction. Instead, we use these as inputs to our model to improve the predictions by using them collectively, instead of independently. ICAF takes the head-based features and speaking probability features as inputs.

\section{ICAF: Iterative Collective Classification}

Here we describe $I C A F$, the collective classification methods that incorporates inter-person dependencies and temporal consistency to jointly predict the VFOA of all people.

Let $\boldsymbol{f}_{i, t}$ denote the raw input feature vector of person $P_{i} \in$ $\left\{P_{1}, \ldots P_{k}\right\}$ at time $t$. The raw input features for $P_{i}$ include the head pose angles vector, the eye gaze vector and speaking probabilities vector $\vec{s}=\left(s_{1}, \ldots, s_{i-1}, 0, s_{i+1}, \ldots, s_{k}\right)$. Note that we don't use $P_{i}$ 's speaking probability $s_{i}$ in $\vec{s}$, as $P_{i}$ 's speaking activity doesn't directly influence her VFOA. Let $C_{i}$ denote the VFOA prediction model for $P_{i}$. ICAF builds separate models $C_{i}$ for each person $P_{i}$. $C_{i}$ outputs a vector $\boldsymbol{v}_{i, t}$, the probability distribution of person $P_{i}$ 's visual focus of attention at time $t$. This output vector specifies the probability that $P_{i}$ 's VFOA is person $P_{j}$ (or the tablet) for each $j$. The ground truth label for person $P_{i}$ at time $t$ is denoted by $y_{i, t}$.

Figure 3 illustrates $I C A F$ for $k$ people and an $L$-layer network. Each person $P_{i}$ has one classifier $C_{i}^{(l)}$ for each layer $l$. Raw features $\boldsymbol{f}_{i, t}$ are used as input for $P_{i}$ at time $t$. The model has multiple layers $1, \ldots, L$ to add inter-person dependencies by using the output of other people's classifiers as input (shown in dotted lines). Each classifier also takes the previous timestep's output as input (shown in dashed lines only for $C_{1}$ for simplicity). The final output vectors are $\boldsymbol{v}_{i, t}^{(L)}$.

$I C A F$ has three major inputs for each classifier $C_{i}^{(l)}$ at every time $t$ and layer $l$ as follows: (i) raw features $\boldsymbol{f}_{i, t}$ associated with $P_{i}$, (ii) inter-person dependencies $\boldsymbol{v}_{j, t}^{(l-1)}(j=$ $1, \ldots, k, j \neq i$ ) incorporating the influence of the behavior of other people, and (iii) temporal consistency $\boldsymbol{v}_{i, t-1}^{(l-1)}$ en- 


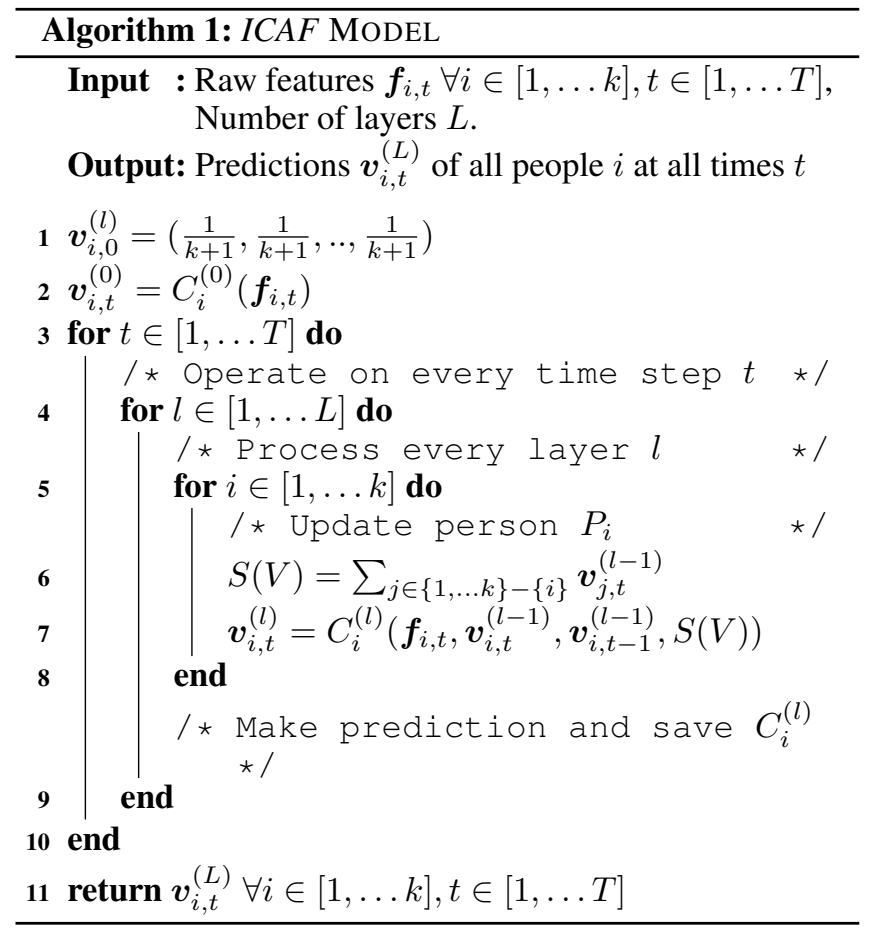

abling the model to make temporally consistent predictions. Together, this results in a collective classification model that makes predictions for all people. The overall algorithm of $I C A F$ is shown in Algorithm 1.

\subsection{Inter-person Dependencies}

In a multi-person discussion, the behavior of one person can influence the VFOA of others. Moreover, the behavior of people is highly correlated-when a person is speaking, other people are likely looking at him [Ba and Odobez, 2011]. This mutual influence can be used to make accurate predictions.

We incorporate the person-to-person influence by adding explicit connections between their classifiers (lines 4-8 in Algorithm 1). In particular, for every person $P_{i}$ 's model $C_{i}$, we use the predictions of all other people's models $C_{j}, \forall j \in$ $\{1, \ldots, k\}-\{i\}$ as input. The resulting model is mutuallyrecursive. To solve this recursion, we unfold the model for multiple layers so that the output of layer $l$ is fed as input to layer $l+1$. This is shown as layers $1, \ldots L$ in Figure 3 .

Thus, the input to person $P_{i}$ 's model $C_{i}^{(l)}$ at layer $l$ is its output from layer $l-1$ and an aggregation of the set $V$ of outputs from other people's models from layer $l-1$. The aggregation is a summation represented as $S(V)$, which is used as an input to the model (lines 6-7 in Algorithm 1).

To initialize for layer 1 , let $\boldsymbol{v}_{i, t}^{(0)}=C_{i}^{(0)}\left(\boldsymbol{f}_{i, t}\right)$, where $C_{i}^{(0)}$ is the classifier trained by only raw features of $P_{i}$, separately.

\subsection{Temporal Consistency}

The VFOA of a person at time $t$ is linked to her VFOA at time $t-1$. The temporal consistency component of ICAF explicitly incorporates this dependency by using the output of the predictions made during the last timestep for the person as an

$$
\boldsymbol{v}_{i, t}^{(l)}=C_{i}^{(l)}(\underbrace{\boldsymbol{f}_{i, t}}_{\text {Raw input }}, \underbrace{\boldsymbol{v}_{i, t}^{(l-1)}}_{\text {Person input Temporal input }}, \underbrace{\boldsymbol{v}_{i, t-1}^{(l-1)}}_{\text {Inter-person input }}, \underbrace{\sum_{j, t} \boldsymbol{v}_{j-1)}^{(l-1)}}_{j \in\{1, \ldots k\}-\{i\}})
$$

Figure 4: Final formulation of ICAF to output $\boldsymbol{v}_{i, t}^{(l)}$ of person $i$ at time $t$ on layer $l$.

input. Specifically, the output $\boldsymbol{v}_{i, t-1}^{(l-1)}$ is an input to $C_{i}^{(l)}$. This is shown using the dashed lines in Figure 3 and in line 7 in Algorithm 1. For each layer $l$, we initialize $\boldsymbol{v}_{i, 0}^{(l)}$ as a uniform probability distribution for VFOA targets.

The final formulation with all the components is shown in Figure 4. Overall, ICAF uses the real time inputs along with temporal and inter-person dependencies to jointly predict the visual focus of attention of all people.

\section{Experiments}

We conduct several experiments on Resistance and AMI datasets to show:

- ICAF outperforms all strong baselines by $1.3 \%$ in predicting VFOA in the next time step (i.e., 10 frames) with $p=0.046$ by two-sample t-test.

- ICAF significantly outperforms the highest baseline by up to $5 \%$ when making predictions upto $k$ time steps in the future $(p<0.05)$.

- Collective classification and temporal dependencies boost the performance of ICAF significantly.

Baselines. We compare with three sets of baselines that use head pose vector $(\mathrm{H})$, eye gaze vector $(\mathrm{E})$, and speaking probability vector(S) for predictions. The first set of baselines are [Ba and Odobez, 2009; Ba and Odobez, 2011; Massé et al., 2017], with comparable numbers of VFOA targets in similar settings. Specifically, $\operatorname{GMM}(\mathrm{H}), \operatorname{GMM}(\mathrm{H}, \mathrm{E})$ use Gaussian Mixture Model with parameters from each individual [Ba and Odobez, 2009]. $\mathrm{HMM}(\mathrm{H}), \mathrm{HMM}(\mathrm{H}, \mathrm{E})$ uses Hidden Markov Model [Ba and Odobez, 2009]. DBN(H,S), DBN(H,E,S) uses Dynamic Bayesian Network (DBN) incorporating conversational dynamics and a shared constant focus prior [Ba and Odobez, 2011]. Note that the screen activity feature is removed to adapt to our dataset. G-DBN uses DBN to track VFOAs and eye gaze simultaneously with people's global head poses as inputs [Massé et al., 2017]. In our dataset, people sit uniformly in a circle, so we convert their local head poses to global ones given poses of their cameras. Further, we created two more sets of baselines using three sets of features $\mathrm{H},(\mathrm{H}, \mathrm{E})$ and $(\mathrm{H}, \mathrm{E}, \mathrm{S})$. The second set of baselines trains one general classifier GC for all people by including the person index as input feature vector [Ba and Odobez, 2011]. The last set of baselines trains a person-specific classifier PC for each person [Asteriadis et al., 2014]. As in the case of GC, we create three baselines $\mathrm{PC}(\mathrm{H}), \mathrm{PC}(\mathrm{H}, \mathrm{E})$, and $\mathrm{PC}(\mathrm{H}, \mathrm{E}, \mathrm{S})$.

Experimental setting. To get speaking probability features, we set the sliding window size as 30 frames (1 sec) and train a Random Forest speaking detection model SP. The training data uses people's introductions as speaking samples, 
Proceedings of the Twenty-Eighth International Joint Conference on Artificial Intelligence (IJCAI-19)

\begin{tabular}{c|c}
\hline GMM(H,E) & 0.716 \\
HMM(H,E) & 0.770 \\
DBN(H,E,S) & 0.800 \\
G-DBN & 0.782 \\
GC(H,E,S) & 0.756 \\
PC(H,E,S) & 0.818 \\
ICAF & $\mathbf{0 . 8 3 1}$ \\
\hline
\end{tabular}

Table 2: Experiment 1: Next VFOA Prediction: Table reports accuracy of $I C A F$ and baselines using all features. Note that the best results of GC, PC, and ICAF are achieved by RF. All improvements of ICAF are statistically significant $(p<0.05)$.

and other people's introductions as non-speaking samples. The introductions were not drawn from our 5 video samples. We evaluate $I C A F$ and baselines by respecting the temporal order of data. Instead of doing a $k$-fold cross-validation, we train the model for the first $T$ data points and test on the $T+1^{\text {th }}$ data point (each data point consists of 10 frames). $T$ is varied from $96.3 \%$ to $99.9 \%$, and the results are averaged. Recall that the data for each game is divided into three parts: an introduction round and two discussion rounds. The introduction round clips are only used for training, and the temporal evaluation is done with the two discussion rounds. Both training and testing are at the frame level. Frame VFOA probabilities are further averaged over 10 frames as probabilities at each 10-frame segments. Given the generality of our model, we experiment with 4 classifiers: Random Forest (RF), Logistic Regression (LR), Linear SVM (LINSVM) and Gaussian Naive Bayes (NB). In all cases, ICAF has 3 layers. All models are compared using the accuracy metric.

Experiment 1: Next VFOA prediction. We compare $I C A F$ with all baselines using all features. All models are trained on the first $T$ data points and then used to predict the $T+1^{\text {th }}$ data point. Note that this means that we are predicting the visual focus of attention for each person $1 / 3$ second into the future. The features given to ICAF for every frame are the head pose vector $(H)$, eye gaze vectors $(E)$, and speaking probability vectors $(S)$. Table 2 shows the results. For fairness, we add eye gaze features $(E)$ to baselines GMM, HMM and DBN. (i) Person-specific baseline models perform better than the corresponding general-classifier baselines using the same set of features. Specifically, PC(H,E,S) performs at least $6.2 \%$ better than $\mathrm{GC}(\mathrm{H}, \mathrm{E}, \mathrm{S})$. (ii) More importantly, ICAF performs between $1.3 \%-11.2 \%$ better than all baseline models. (iii) Indeed, it is 3\% higher than state-ofthe-art method DBN(H,E,S).

Experiment 2: Longer-future predictions. We next evaluate the robustness of $I C A F$ by predicting the $T+k^{t h}$ data point while training only till the $T^{t h}$ data point. We vary $k$ from 1 to 10 , meaning that we predict who a person will look at between 0.3 and 3.3 seconds into the future. Figure 5 shows the result. ICAF outperforms the best baseline by up to $5 \%$. In fact, it is better than $\mathrm{DBN}(\mathrm{H}, \mathrm{E}, \mathrm{S})$ by $1.5 \%-5.7 \%$. Moreover, ICAF is relatively stable as $k$ increases, while some baselines drop rapidly. Specifically, ICAF's prediction accuracy varies only $7.5 \%$ over $k$, so it gives robust estimation of VFOA in the longer-term future.

Experiment 3: Contribution of collective classification. Figure 6 compares the results of $I C A F$ with and without the

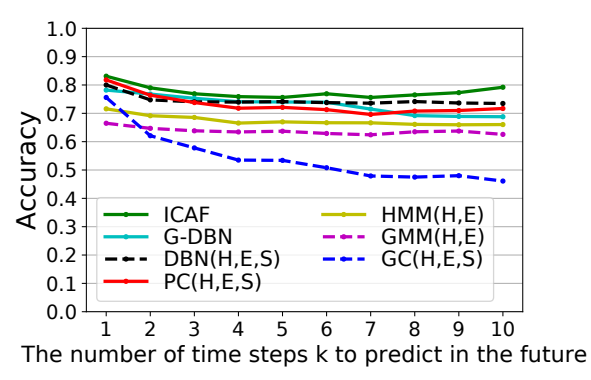

Figure 5: Experiment 2: Longer-Future Prediction: Accuracy of predicting $\mathrm{k}$ steps to the future. ICAF is the highest over all time steps, and outperforms the best baseline by up to $5 \%(p<0.05)$.

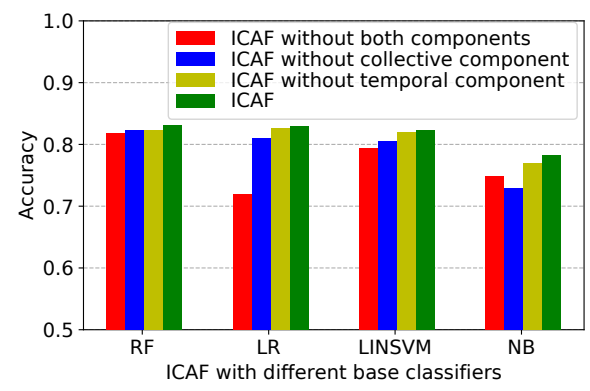

Figure 6: Experiment 3: Contribution of collective classification: The performance drops when either the collective or the temporal components is removed and drastically when both are removed.

temporal and collective classification components. Note that $I C A F$ without both components is equivalent to the baseline $\mathrm{PC}(\mathrm{H}, \mathrm{E}, \mathrm{S})$. We observe that each of them boost the performance of ICAF from $0.2 \%$ to $5.3 \%$ w.r.t. all base classifiers. The combination of both components is important in $I C A F$ : the performance of $\mathrm{PC}(\mathrm{H}, \mathrm{E}, \mathrm{S})$ is lower than $I C A F$ without either of the components. Additionally, adding collective classification improves performance more than the temporal component alone. Therefore, both temporal and collective classification components of ICAF are essential, and the collective component results is more critical for good predictions.

Experiment 4: Comparison with different features. We next explore the effects of different features on ICAF and baselines. Note that RF is used as the (base) classifier to obtain best results for GC, PC, and ICAF . Table 3 shows the results for next VFOA prediction. First, for all models, eye gaze features $\mathrm{E}$ boost the predictions. It especially boosts [ $\mathrm{Ba}$ and Odobez, 2011; Ba and Odobez, 2009] by at least $13.5 \%$. Second, speaking features $\mathrm{S}$ boost all models except for GC. These demonstrate that both $\mathrm{E}$ and $\mathrm{S}$ contribute to prediction of VFOA. Third, using features including $E$ or $S, I C A F$ outperforms all baselines.

Experiment 5: Comparison between different base classifiers. Here we explore performance of $I C A F$ with different kinds of base classifiers: RF, LR, NB and LINSVM. In Figure 7 we compare ICAF with $\mathrm{GC}$ and $\mathrm{PC}$ in the cases of both next VFOA prediction $(k=1)$ and longer-future VFOA prediction $(k>1)$. We only show 2 out of 4 plots due to space limit, but the results are similar. The colored texts show the 
Proceedings of the Twenty-Eighth International Joint Conference on Artificial Intelligence (IJCAI-19)

\begin{tabular}{c|c|c|c|c}
\hline Model & $\mathrm{H}$ & $\mathrm{H}, \mathrm{E}$ & $\mathrm{H}, \mathrm{S}$ & $\mathrm{H}, \mathrm{E}, \mathrm{S}$ \\
\hline GMM & 0.525 & 0.716 & - & - \\
HMM & 0.623 & 0.770 & - & - \\
DBN & - & - & 0.665 & 0.800 \\
GC & $\mathbf{0 . 7 1 9}$ & 0.799 & 0.731 & 0.756 \\
PC & 0.716 & 0.805 & 0.771 & 0.818 \\
ICAF & 0.718 & $\mathbf{0 . 8 1 1}$ & $\mathbf{0 . 7 8 4}$ & $\mathbf{0 . 8 3 1}$ \\
\hline
\end{tabular}

\begin{tabular}{c|c|c}
\hline Model & Static meetings & Dynamic meetings \\
\hline [Ba and Odobez, 2011] & 0.556 & 0.520 \\
ICAF & $\mathbf{0 . 5 6 8}$ & $\mathbf{0 . 5 3 8}$ \\
\hline
\end{tabular}

Table 4: AMI corpus experiments. Accuracy of the proposed model on static and dynamic meetings.

Table 3: Experiment 4: Comparison between different features: Both $\mathrm{E}$ and $\mathrm{S}$ boost the accuracy of all models except $\mathrm{GC}$, and $I C A F$ performs the best in 3 out of 4 cases. $(p<0.05)$

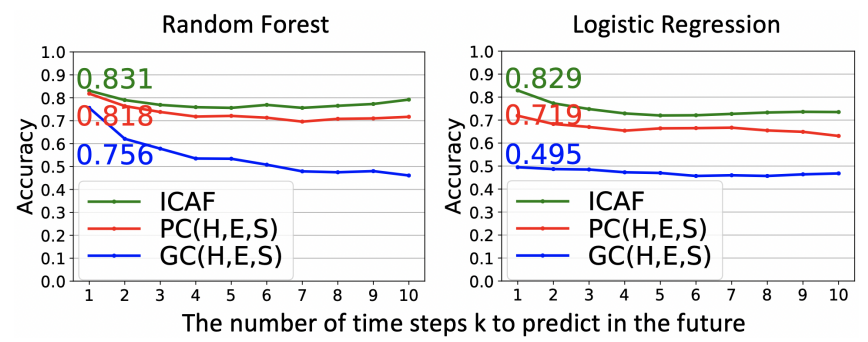

Figure 7: Experiment 5: Comparison between different (base) classifiers. In each subfigure, each of 3 colored numbers indicates the prediction accuracy of $k=1$ in the same colored line.

results for $k=1$, where $I C A F$ outperforms the corresponding best baseline by $1.3 \%-11 \%$. For $k>1$, it outperforms the best baseline by up to $5 \%$ with RF, $12 \%$ with LR, $3 \%$ with LINSVM, and $4 \%$ with NB. Thus, we observe the generality of ICAF.

AMI corpus experiments. We also conducted experiments on the AMI meeting corpus [McCowan et al., 2005]. 8 meetings are dynamic, where people sit around a table and upto 1 person moves to the whiteboard/screen to present. 4 meetings are static, where all people remain seated. We use people's closeup videos to extract head pose, eye gaze, and speaking probability. We followed the leave-one-out protocol as in [Ba and Odobez, 2011] and compare frame-based accuracy. Since the 4 seats over all meetings are fixed, we train seat-specific classifiers in ICAF. Table 4 shows that ICAF outperforms [Ba and Odobez, 2011] in both kinds of meetings.

\section{Lightly Supervised VFOA Prediction}

A major challenge in VFOA prediction is the lack of labeled data for new videos. Annotating VFOA at a second or subsecond granularity is highly time-consuming and often not clean. We now propose to generate accurate VFOA predictions without ground truth labels. The proposed technique is general and can be used to train both the baselines and $I C A F$.

The intuition is that people are highly likely to look at the person who is speaking if there is a single speaker [Stiefelhagen et al., 2002]. Building on this intuition, we identify continuous clip segments where one person is speaking. This is done using the speaking prediction model SP described in Section 3.1. To reduce false positives, we further average over 10 frames' prediction probability around the current frame and use it as the final label to select single-speaker segments. For a segment where $P_{i}$ is speaking, we assign $i$ as the training label for all other people and the model is trained with it. To evaluate the effectiveness of this training method, we train

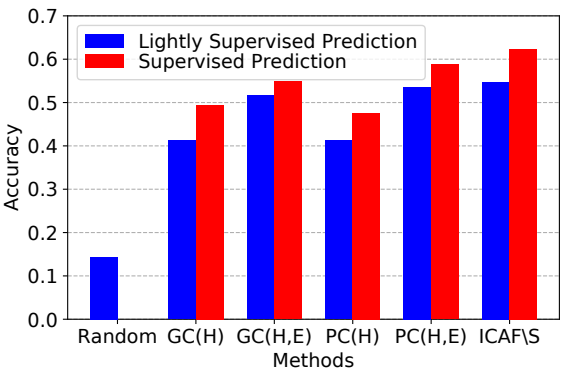

Figure 8: Lightly supervised predictions (in blue) and supervised predictions (in red): 'Random' denotes random prediction accuracy, and $I C A F \backslash \mathrm{S}$ denotes $I C A F$ without speaking feature.

all models using the introduction (by generating its speaker labels) and use the two discussion clips with the ground truth VFOA labels as test.

Figure 8 shows the results for all baselines and ICAF using $\mathrm{RF}$ as base classifier. Since the training labels are speaking labels, we remove speaking probability features from ICAF as well as baselines. Compared to random prediction of $14.4 \%$, the lightly supervised training technique generates $41.2 \%$ $54.7 \%$ results. We also observe that ICAF performs better than the baselines. For comparison, Figure 8 shows the equivalent result with supervised training, where we train the models using the ground truth focus labels in the introduction round as well. We note that the lightly supervised prediction is comparable to supervised prediction, showing the effectiveness of the proposed training technique.

\section{Conclusion}

We showed that by explicitly incorporating inter-person dependencies and temporal consistency are crucial to accurately predict VFOA both in short-term future and long-term future. The ICAF model is, therefore, able to overcome the challenges of rapidly changing VFOA, high dynamics of the discussion, and person-person inter-dependencies. Moreover, the lightly supervised ICAF is crucial in making the model general to unseen videos. This opens doors to new research in efficient extraction of interaction networks from videos without any training labels.

Role of Authors. Authors Metzger and Nunamaker designed the Resistance-style game and collected the Resistance data. The remaining authors designed the machine learning algorithms and software, and designed/ran all experiments.

\section{Acknowledgements}

This work was funded in parts by ARO Grant W911NF1610342, NSF OAC-1835598, DARPA MCS, ARO MURI, JD.com, Amazon, and Stanford Data Science Initiative. JL is a Chan Zuckerberg Biohub investigator. 


\section{References}

[Asteriadis et al., 2014] Stylianos Asteriadis, Kostas Karpouzis, and Stefanos Kollias. Visual focus of attention in non-calibrated environments using gaze estimation. International Journal of Computer Vision, 107(3):293-316, 2014.

[Ba and Odobez, 2008] Sileye O Ba and Jean-Marc Odobez. Multi-party focus of attention recognition in meetings from head pose and multimodal contextual cues. In Acoustics, Speech and Signal Processing, 2008. ICASSP 2008. IEEE International Conference on, pages 22212224. IEEE, 2008.

[Ba and Odobez, 2009] Sileye O Ba and Jean-Marc Odobez. Recognizing visual focus of attention from head pose in natural meetings. IEEE Transactions on Systems, Man, and Cybernetics, Part B (Cybernetics), 39(1):16-33, 2009.

[Ba and Odobez, 2011] Sileye O Ba and Jean-Marc Odobez. Multiperson visual focus of attention from head pose and meeting contextual cues. IEEE Transactions on Pattern Analysis and Machine Intelligence, 33(1):101-116, 2011.

[Baltrusaitis et al., 2018] T. Baltrusaitis, A. Zadeh, Y. C. Lim, and L. P. Morency. Openface 2.0: Facial behavior analysis toolkit. In 2018 13th IEEE International Conference on Automatic Face Gesture Recognition (FG 2018), pages 59-66, May 2018.

[Bilgic et al., 2007] Mustafa Bilgic, Galileo Mark Namata, and Lise Getoor. Combining collective classification and link prediction. In Data Mining Workshops, 2007. ICDM Workshops 2007. Seventh IEEE International Conference on, pages 381-386. IEEE, 2007.

[Duffner and Garcia, 2013] Stefan Duffner and Christophe Garcia. Unsupervised online learning of visual focus of attention. In Advanced Video and Signal Based Surveillance (AVSS), 2013 10th IEEE International Conference on, pages 25-30. IEEE, 2013.

[Duffner and Garcia, 2016] Stefan Duffner and Christophe Garcia. Visual focus of attention estimation with unsupervised incremental learning. IEEE Transactions on Circuits and Systems for Video Technology, 26(12):22642272, 2016.

[Hall et al., 2005] Judith A Hall, Erik J Coats, and Lavonia Smith LeBeau. Nonverbal behavior and the vertical dimension of social relations: a meta-analysis. Psychological bulletin, 131(6):898, 2005.

[Knapp et al., 2013] Mark L Knapp, Judith A Hall, and Terrence G Horgan. Nonverbal communication in human interaction. Cengage Learning, 2013.

[Kong et al., 2012] Xiangnan Kong, Philip S Yu, Ying Ding, and David J Wild. Meta path-based collective classification in heterogeneous information networks. In Proceedings of the 21st ACM international conference on Information and knowledge management, pages 1567-1571. ACM, 2012.
[Massé et al., 2017] Benoît Massé, Silèye Ba, and Radu Horaud. Tracking gaze and visual focus of attention of people involved in social interaction. IEEE Transactions on Pattern Analysis and Machine Intelligence, 2017.

[McCowan et al., 2005] Iain McCowan, Jean Carletta, W Kraaij, S Ashby, S Bourban, M Flynn, M Guillemot, T Hain, J Kadlec, V Karaiskos, et al. The ami meeting corpus. In Proceedings of the 5th International Conference on Methods and Techniques in Behavioral Research, volume 88, page 100, 2005.

[Rayner, 2009] Keith Rayner. Eye movements and attention in reading, scene perception, and visual search. The quarterly journal of experimental psychology, 62(8):14571506, 2009.

[Sen et al., 2008] Prithviraj Sen, Galileo Namata, Mustafa Bilgic, Lise Getoor, Brian Galligher, and Tina Eliassi-Rad. Collective classification in network data. AI magazine, 29(3):93, 2008.

[Sheikhi and Odobez, 2012] Samira Sheikhi and Jean-Marc Odobez. Investigating the midline effect for visual focus of attention recognition. In Proceedings of the 14th ACM international conference on Multimodal interaction, pages 221-224. ACM, 2012.

[Stiefelhagen and Zhu, 2002] Rainer Stiefelhagen and Jie Zhu. Head orientation and gaze direction in meetings. In CHI'02 Extended Abstracts on Human Factors in Computing Systems, pages 858-859. ACM, 2002.

[Stiefelhagen et al., 1999] Rainer Stiefelhagen, Michael Finke, Jie Yang, and Alex Waibel. From gaze to focus of attention. In International Conference on Advances in Visual Information Systems, pages 765-772. Springer, 1999.

[Stiefelhagen et al., 2002] Rainer Stiefelhagen, Jie Yang, and Alex Waibel. Modeling focus of attention for meeting indexing based on multiple cues. IEEE Transactions on Neural Networks, 13(4):928-938, 2002.

[Taskar et al., 2004] Ben Taskar, Ming-Fai Wong, Pieter Abbeel, and Daphne Koller. Link prediction in relational data. In Advances in neural information processing systems, pages 659-666, 2004.

[Voit and Stiefelhagen, 2008] Michael Voit and Rainer Stiefelhagen. Deducing the visual focus of attention from head pose estimation in dynamic multi-view meeting scenarios. In Proceedings of the 10th international conference on Multimodal interfaces, pages 173-180. ACM, 2008.

[Zhang et al., 2008] Honggang Zhang, Lorant Toth, Jun Guo, Jie Yang, et al. Monitoring visual focus of attention via local discriminant projection. In Proceedings of the 1st ACM international conference on Multimedia information retrieval, pages 18-23. ACM, 2008. 